\title{
3D dynamical structuring of a high latitude erupting prominence: II- Analysis of the coronal context and eruption
}

\author{
Serge Koutchmy ${ }^{1}$, Boris Filippov ${ }^{2}$, Ehsan Tavabi ${ }^{3}$, Cyrille Bazin $^{1}$ and \\ Sylvain Weiller ${ }^{1}$ \\ ${ }^{1}$ IAP UMR 7095, INSU-CNRS and UPMC, 98 bis Bd Arago 75014 Paris, France \\ email: koutchmy@iap.fr, bazin@iap.fr, sweiller@free.fr \\ ${ }^{2}$ Pushkov Institute of Terrestrial Magnetism, Ionosphere and Radio Wave Propagation, \\ Russian Academy of Sciences, Troitsk, Moscow Region 142190, Russia \\ email: boris_filippov@mail.ru \\ ${ }^{3}$ Payame Noor University of Tehran, 14155-6466,I.R. of Iran \\ email: etavabi@gmail.com
}

\begin{abstract}
Both the origin of the quiescent prominences and their eruption related to CMEs event are still a matter of extended studies. The case of high latitudes quiescent prominences producing slow CMEs can be considered as a potential component of the slow wind. A high latitude prominence was observed on November 13 - 14, 2011. A schematic representation of flux rope is proposed to describe the magnetic structure of the prominence prior to its eruption.
\end{abstract}

Keywords. prominence eruption, flows, counter- flows, vortices, tornado, CME

\section{Observations}

A high latitude prominence (see Fig. 1 of Koutchmy 2013a this issue) was observed on November 13 - 14, 2011, using high resolution $\mathrm{H} \alpha$ fast imaging.

Prior to the prominence eruption, radial counter-flows with vortices are observed in threads with a dominant downward motion of plasma blobs or plasmoids. Some blobs might move along field lines and at the same time could be seen as moving vertically. However, we cannot exclude that some blobs might fall through the dominantly horizontal field under the action of gravity and due to plasma instabilities. We primarily used SDO/AIA coronal and transition region (TR) filtergrams processed to improve the signal/noise ratio, see Fig. 1 left panel, and SoHO/LASCO images, to look at the details of the erupting southern high-latitude prominence on 14 - 15 November 2011 and the corresponding very low speed CME event $(\mathrm{v}=163 \mathrm{~km} / \mathrm{s}$ from LASCO data processed at NRL). The intermingled cool chromospheric and TR details (in white in Fig. 1) with the hot more stretched coronal details (in dark in Fig. 1) are particularly striking, in apparent contrast with the usual picture of a cavity surrounding the prominences. The prominence is here a part of a southern polar crown filament which is too faint and possibly too high to derive its chirality from the observed internal fine structure as it is usually possible for active region filaments, although internal motions observed within the prominence in $\mathrm{H} \alpha$ suggest negative helicity of the flux rope, which is not unusual but not typical for the southern hemisphere. However, the prominence is not stretched strictly in longitudinal direction but turns to the North in its left-hand side, see Fig. 1 right panel. Therefore, we believe the prominence is seen from the Earth at the angle about $45^{\circ}$ to its long axis or direction of the polarity inversion line. 

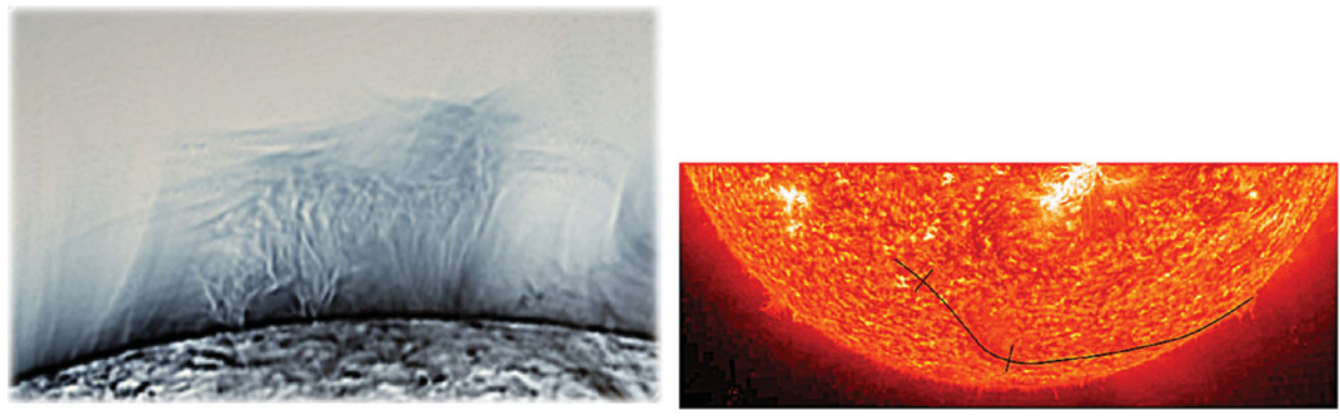

Figure 1. (left panel) Negative processed coronal image from a superposition of 200 elementary images taken with the FeXII 195 filter of AIA/SDO during our observations. (right panel) Filament channel (black line) deduced from the STEREO/B 304 SECCHI images on 01-15 November 2011 drawn on the image on 11 November at 06 UT.

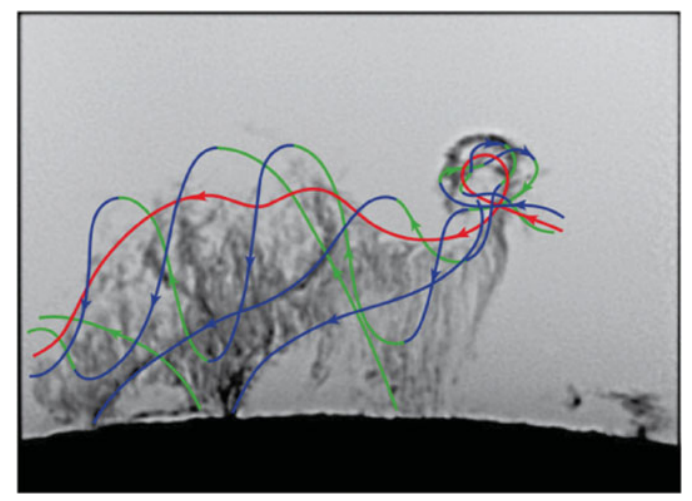

Figure 2. Negative $\mathrm{H} \alpha$ image of the prominence on 14 November with schematic representation of flux-rope helical field lines. Parts of the field lines located before (behind of) the prominence symmetry plane have blue (green) color. Red line shows the flux-rope axis or the filament spine.

\section{Results}

A 3D structure is proposed to represent the prominence (Fig. 2). The details of the eruption are analyzed using additional SECCHI (STEREO mission of NASA) simultaneous filtergrams, see Fig. 1 right panel and the LASCO coronagraph data. The magnetic context is discussed, including the use of a computed PFSS map, although photospheric magnetic fields patches inferred from HMI magnetograms of SDO are rather weak near the filament location and do not show a signature. A likely scenario is suggested taking into account the 3D peculiarities for explaining this very low speed CME.

\section{References}

Engvold, et al. 1989, Hvar Obs. Bull., 13, 205

Filippov, B. \& Koutchmy, S. 2008, Ann. Geophys., 26, 3025

Koutchmy, S. Filippov, B. Tavabi, E. Weiller, S., \& Bazin, C. 2013, IAUS-300 Proc. Poster I Regnier, S. Walsh, R. N., \& Alexander, C. E. 2011, Astron. Astrophys., 533, L1 